by drawing a large ' $\mathrm{X}$ ' from the four corners of the state and placing the university at the centre of it - in order to make it equally accessible to all students.

The University of Pittsburgh (Pitt) is a public university that resides, as one might have guessed, in Pittsburgh, the second largest city in Pennsylvania and at the western edge of the state.

Douglas J. Jerolmack

Department of Earth and Environmental Science, University of Pennsylvania, Hayden Hall,

240 South 33rd Street, Philadelphia,

Pennsylvania 19104, USA

\section{Animal welfare is not just another bureaucratic hoop}

SIR - I disagree with C. Jimenez's reply, in Correspondence, opposing Victoria Buck's suggestion of making animal-welfare sections in scientific papers compulsory (Animal-welfare section in papers would be a burden' Nature 447, 259; 2007). We all have a great many bureaucratic hoops to jump through these days, but we should not take a dismissive attitude to animal-welfare issues.

Animal-rights extremists have made life a misery for some scientists in the United Kingdom, despite our having one of the best-regulated licensing and ethical review processes in the world. National legislation requires scientists wishing to carry out experiments on animals to be licensed, and strict enforcement by both the legislature and by the local ethical review committees ensures that there are very few infringements.

The exchange between Buck and Jimenez did not address ethical approval statements, but for the record I do not think it an onerous task to include in scientific papers a paragraph stating the legislation(s) and local ethical review process under which the work had been approved. Many journals, including the Nature journals, already make compliance a condition of publication (see www.nature.com/authors/editorial_policies/ experimental.html).

Although we must be robust in our defence of the need for appropriate animal experimentation, it is pointless to antagonize those individuals who will never be persuaded of its need or relevance. The 3 Rs requirement goes some way to assuaging the disquiet of the more reasonable objectors, and hence should not be dismissed.

We live in a cynical world where everything is questioned, and the scientist is no longer seen as an ivory-tower figure. We are all accountable to the agencies that fund us and regulate our use of experimental animals and human tissue samples.

Our ability to pursue science gives us a privilege that few others enjoy, that of unravelling the biological processes that make us what we are. We are enabled in this occupation by the silent consensus, and hope, of people all over the world. We abuse that consensus at our peril.

L. Bergmeier

University of London, London, UK

\section{Animal welfare: reporting details is good science}

SIR - C. Jimenez, in Correspondence, considers that detailed information on the way animals are handled and treated should not be placed in published papers (Nature 447, 259; 2007).

I disagree, because it is a fundamental principle of the scientific process that when a paper is published, the study can be repeated from the description given in the methods, thereby allowing external validity to be assessed. To this end, variables that might affect the results need to be reported accurately.

It is well-documented that making even a slight change to a laboratory animal's environment or husbandry can have profound influences on its biological functioning. Cage size can influence metabolism, baseline rectal temperature, the fever response, feeding behaviour and behavioural responses in predator-prey interactions. The type of flooring in a cage can affect blood pressure, heart rate and body temperature. Other factors that influence physiology and behaviour include housing laboratory mice as singletons or pairs, the complexity of the cage and the extent to which animals are handled.

Variables such as these, which might be changed to improve the welfare of the animals, should be reported in published papers as an essential component of the accurate reporting of science.

\section{M. Sherwin}

University of Bristol, Bristol, UK

\section{UNAIDS rejects claims of exaggeration and bias}

SIR - We would like to provide our perspective on your Book Review of two books criticizing the Joint United Nations Programme on AIDS (UNAIDS), 'Time for a change?' (Nature 447, 531-532; 2007), and the coverage of this issue at www.nature.com/ news/2007/070528/full/070528-6.html.

In his book The AIDS Pandemic: The Collision of Epidemiology with Political Correctness, James Chin accuses UNAIDS of exaggerating data for the sake of advocacy, which is not true. Nor are UNAIDS data influenced by political or fundraising agendas. The UNAIDS Secretariat and the World Health Organization work closely with other technical partner organizations to assist countries in better understanding their HIV epidemics so they can respond appropriately. Estimations are produced in close collaboration with national epidemiologists and governments, using methodologies recommended by an international team of experts chaired by a leading academic from Imperial College London.

UNAIDS is committed to providing the most accurate information available and continues to be transparent in publicizing the methods used to assess the magnitude of the past and current epidemics. UNAIDS has always stated that countries should use the most comprehensive and most recent data available. Reassessments of earlier published estimates of prevalence, incidence and mortality have been made, and we expect that there may be adjustments in the future.

Helen Epstein's The Invisible Cure also makes inaccurate statements about the work of UNAIDS: in particular, we have always advocated the reduction of number of sexual partners as an effective strategy for HIV prevention, as can be seen from our reports and other contributions to the published record. All UNAIDS documents on the prevention of sexual transmission of HIV advocate abstinence, reduction of sexual partners and correct use of male and/or female condoms. (See, for example, http:// data.unaids.org/Global-Reports/Bangkok2004/UNAIDS_Bangkok_press/GAR2004_ pdf/GAR2004_ExecSumm_en.pdf)

UNAIDS and its partners will continue their mission to gather the best-quality data to assist in shaping an effective global response to AIDS.

Paul R. De Lay ${ }^{\star}$, Kevin M. De Cock $\dagger$

*Evidence, Monitoring and Policy, UNAIDS, 20 Avenue Appia, 1211 Geneva, Switzerland $\dagger$ HIV Department, World Health Organization, 19 Avenue Appia, 1211 Geneva, Switzerland

\section{Chinese recorded classical nova two millennia ago}

SIR - I agree with Michael M. Shara and colleagues (Nature 446, 159-162; 2007) that the star Z Camelopardalis was a classical nova a few thousand years ago. In fact, a record of the eruption exists in Chinese documents of the time. There was, apparently, a report of a 'guest star' in October-November 77 в (P. Y. Ho Vistas Astron. 5, 127-225; 1962). The position in the sky fits Z Camelopardalis. This seems to be the oldest classical nova recorded in any surviving text.

Göran H. I. Johansson

Tordönsvägen 4G, 1tr,

SE-22227 Lund, Sweden 